Results 33 children (45 eyes) underwent cataract surgery during the 2 year study period. Only 10 were referred following abnormal NIPE and fewer than 50\% were referred before 9 weeks of age. Of the 90 normal newborns examined at the first NIPE check, visually insignificant congenital media opacities were detected in $9(10 \%)$ infants on CatCam imaging alone. CatCam examination was subjectively easier than red reflex testing, particularly in non-Caucasian infants. Finally, 111 subjects attending a specialist clinic were examined with DO and CatCam prior to pupil dilatation and specialist review. The sensitivity and specificity for media opacity was $100 \%$ for CatCam and $71 \%$ and $62 \%$ respectively for the DO $(\mathrm{p}=0.01)$.

Conclusion Although some cataracts may have developed postnatally, our audit suggests that the sensitivity of the NIPE examination is poor and that many infants with cataracts are diagnosed late. The clinical studies demonstrate the advantages of CatCam over DO examination, particularly its high sensitivity and specificity due to the absence of pupil constriction, better reflectivity of infrared light from non-Caucasian fundi and ability to document the images facilitating a second opinion.

\section{G192 C-REACTIVE PROTEIN FOR DIAGNOSING LATE-ONSET INFECTION IN NEWBORN INFANTS: COCHRANE REVIEW OF TEST ACCURACY}

${ }^{1}$ JVE Brown, ${ }^{1} \mathrm{~N}$ Meader, ${ }^{1,2}$ J Cleminson, ${ }^{1} \mathrm{~W}$ McGuire. ${ }^{1}$ Centre for Reviews and Dissemination, University of York, York, UK; ${ }^{2}$ Newcastle Neonatal Service, Newcastle Hospitals NHS Foundation Trust, Newcastle upon Tyne, UK

\subsection{6/archdischild-2018-rcpch.187}

Aims To determine the accuracy of elevated serum C-reactive protein (CRP) for diagnosing late-onset neonatal infection.

Methods Cochrane systematic review of diagnostic test accuracy. We searched MEDLINE, Embase, and Science Citation Index to September 2017 for cohort and cross sectional studies evaluating the diagnostic accuracy of serum CRP for detecting late-onset infection in newborns:

Index test: Serum CRP level (threshold defined by individual studies).

Reference standards Invasive infection diagnosed $>72$ hour after birth, confirmed by culture from a normally sterile site or findings on autopsy examination consistent with invasive microbial infection.

We screened titles and abstracts and evaluated the full text of possibly eligible articles. We extracted study characteristics and used the Quality Assessment of Diagnostic Accuracy Studies-2 (QUADAS-2) tool to assess quality. One reviewer extracted data for calculation of diagnostic accuracy parameters. These were checked independently by a second reviewer with referral to a third reviewer to resolve discrepancies. We constructed 'two-by- two' tables based on data from the reference standard and index test and created forest plots for sensitivity and specificity. We conducted a bivariate random effects metaanalysis of sensitivity and specificity data and used these estimates to construct a summary receiver operating characteristic curve. We estimated post-test probabilities of late-onset neonatal sepsis based on a range of pre-test probabilities.

Results We included 20 studies (total number of infants 1,615) with sample sizes ranging between 11 and 184. Most studies were conducted in high income countries, investigating both term and preterm babies. Overall, the methodological quality of the studies was good and the risk of bias low.

Data synthesis Pooled sensitivity: 0.58 (95\% CI 0.45 to 0.69 ); Pooled specificity: 0.79 (95\% CI 0.69 to 0.86). There was relatively high heterogeneity as reflected in the forest plots and 95\% prediction region.

Positive likelihood ratio: 2.73 (95\% CI 1.95 to 3.84); Negative likelihood ratio 0.54 (95\% CI 0.42 to 0.69 ).

Conclusion Meta-analysis shows that diagnostic accuracy of serum CRP level is modest. Serum CRP level in this context is not sufficiently accurate to reliably confirm or exclude a diagnosis of infection.

\section{G193 THE TRAIN STUDY: TRANSFUSION IN NEONATES AND IDEAL RED CELL VOLUME STUDY, A RANDOMISED CONTROL TRIAL: ISRCTN68861901}

${ }^{1} S$ Elmusharaf Abdelrahman, ${ }^{1} \mathrm{M}$ Bahari, ${ }^{1} \mathrm{~A}$ Mareri, ${ }^{1,2} \mathrm{R}$ Segurado, ${ }^{3} \mathrm{~J}$ Quigley, ${ }^{3} \mathrm{M}$ Culliton, 3J Fitzpatrick, ${ }^{1} \mathrm{~B}$ Paturi, ${ }^{1} \mathrm{C}$ Vavasseur, ${ }^{1,2,4,5,6} \mathrm{E}$ Molloy. ${ }^{1}$ Paediatrics, National Maternity Hospital, Dublin, Ireland; '2School of Medicine and Medical Sciences, CSTAR, University College Dublin, Dublin, Ireland; ${ }^{3}$ Haematology, National Maternity Hospital, Dublin, Ireland; ${ }^{4}$ Neonatology, Our Lady's Children's Hospital, Crumlin, Dublin, Ireland; ${ }^{5}$ Academic Paediatrics, Trinity College Dublin, National Children's Hospital, Tallaght, Dublin, Ireland;

${ }^{6}$ Paediatrics, Coombe Women's and Infant's University Hospital, Dublin, Ireland

\subsection{6/archdischild-2018-rcpch. 188}

Aim There are different guidelines to calculate red blood cell (RBC) replacement volume in neonates, ranging from $5 \mathrm{ml} / \mathrm{kg}$ up to $20 \mathrm{ml} / \mathrm{kg} \mathrm{RBC}$ volume to be transfused. We aimed to investigate which method is more reliable in achieving the desired Haemoglobin $(\mathrm{Hb})$ from a single blood transfusion in infants $<32$ weeks gestation admitted to the Neonatal Intensive Care Unit (NICU).

Methods Preterm infants $<32$ weeks gestations were enrolled if they were admitted to the Neonatal Intensive Care Unit, required a RBC transfusion and parental consent was obtained. Infants were excluded if there was evidence of active bleeding, intraventricular haemorrhage (IVH) grade $\geq$ III or more at the time of transfusion, $<24$ hours post surgical intervention, $\mathrm{ABO} / \mathrm{Rh}$ incompatibility or Disseminated Intravascular Coagulopathy. Each infant was then randomised to either the standard practice of calculating $\mathrm{RBC}$ volume ( $\mathrm{RBC}$ volume $=20 \mathrm{ml} / \mathrm{kg}$ ) or to the intervention volume calculation $(\mathrm{RBC}$ volume $=5 \times$ working weight $\times[\mathrm{Hb}$ desired $-\mathrm{Hb}$ current]).

Results Sixty three infants were randomised, 55 infants had values for both the post-transfusion $\mathrm{Hb}$ and the target $\mathrm{Hb}$. A chi-square test was used to determine if there was an association between the group to which the infant was randomised and whether they achieved the target $\mathrm{Hb}$ level. 21 (84.0\%) of the 25 infants in the control group achieved the target $\mathrm{Hb}$ level, and $20(66.7 \%)$ of the 30 infants in the intervention group achieved the target $\mathrm{Hb}$ level. Testing at a $5 \%$ significance level, there is no significant difference between the control and intervention groups in the proportion of infants who achieved the target $\mathrm{Hb}$ level $(\mathrm{p}=0.142, \mathrm{df}=1)$.

Conclusion There was no significant difference between the 2 methods of RBC volume calculation in achieving the target $\mathrm{Hb}$. The simpler calculation method of $20 \mathrm{ml} / \mathrm{kg}$ may be the optimum method as less chance of calculation error. 